\title{
Evaluation of Intra-Articular Administration of Platelet-Rich Plasma in the Treatment of Knee-Joint Osteoarthritis
}

\author{
S. Roy ${ }^{1}$, B.K. Bandyopadhyay², D. Bhattacharya ${ }^{3}$, P. Biswas ${ }^{4}$ \\ ${ }^{1}$ Department of Anaesthesiology, Critical Care and Pain, R. G. Kar Medical College, Kolkata, West Bengal, India. \\ ${ }^{2}$ Department of Anaesthesiology, Critical Care and Pain, R. G. Kar Medical College, Kolkata, West Bengal, India. \\ ${ }^{3}$ Department of Anaesthesiology, Critical Care and Pain, R. G. Kar Medical College, Kolkata, West Bengal, India. \\ ${ }^{4}$ Department of Anaesthesiology, Raiganj Medical College, Uttar Dinajpur, West Bengal, India.
}

\section{ABSTRACT}

\section{BACKGROUND}

Knee osteoarthritis $(\mathrm{OA})$ is a very common progressive articular disease usually affecting old age people. Current treatments for knee osteoarthritis are mostly palliative and attack the symptoms rather than influencing the biochemical environment of the joint. Platelet-Rich Plasma (PRP) therapy is being used recently to prevent the progress of the disease in addition to palliation of symptoms. The aim of this interventional study was to assess the effect of intra-articular injection of platelet-rich plasma in 100 patients suffering from knee joint osteoarthritis, attending our pain clinic.

\section{METHODS}

A prospective interventional study was undertaken on 100 patients of American Society of Anaesthesiologist (ASA) Physical Status 1, 2 and 3, aged 30-80 years, suffering from knee joint osteoarthritis Grade I, II and III using the KellgrenLawrence radiographic classification scale, attending the pain clinic who did not respond to pharmacological or physical therapy for more than three months. Visual Analogue Scale (VAS) Score was used to assess the intensity of pain and Western Ontario and McMaster Universities Arthritis Index (WOMAC) Score was used to assess pain, stiffness and physical function of the joint before and after intraarticular injection of PRP at 2,4,8 and 12 weeks.

\section{RESULTS}

The results showed improvement in both the scores from $2^{\text {nd }}$ week onwards itself after injection of PRP in all groups. Grade I and II groups showed significant improvement compared to Grade III at 12 weeks, $\mathrm{p}<0.01$.

\section{CONCLUSIONS}

Intra-articular PRP is effective and safe treatment to relieve pain and disability in patients suffering from knee joint osteoarthritis.

\section{KEY WORDS}

Knee Joint Osteoarthritis, Kellgren-Lawrence Radiographic Classification, PlateletRich Plasma, VAS Score, WOMAC Score
Corresponding Author: Dr. Bijoy Kumar Bandyopadhyay, Srijain Midlands Block 1,

Flat 2D, 83 Jessore Road (South),

Post Office-Ganganagar,

North 24 Parganas, Kolkata-700132,

West Bengal, India.

E-mail: bjoybnrjee@gmail.com

DOI: $10.14260 /$ jemds/2019/735

Financial or Other Competing Interests: None.

How to Cite This Article:

Roy S, Bandyopadhyay BK, Bhattacharya $D$, et al. Evaluation of intra-articular administration of platelet- rich plasma in the treatment of knee-joint osteoarthritis. J. Evolution Med. Dent. Sci. 2019;8(45): 3387-3391, DOI:

10.14260/jemds/2019/735

Submission 27-08-2019,

Peer Review 24-10-2019,

Acceptance 30-10-2019,

Published 11-11-2019. 


\section{BACKGROUND}

Knee osteoarthritis (OA) is a chronic progressive disease affecting more than $20 \%$ of people older than 45 years. ${ }^{1}$ Men are more often affected than women in populations younger than 50 years. Beyond 65 years of age, however, women are affected twice as much as men. ${ }^{2}$ OA Knee is the second most common cause of loss of work performance after low back pain. ${ }^{3}$

Osteoarthritis $(\mathrm{OA})$ is a complex degenerative disease affecting all compartments of the joint. Though it is a degenerative disease, inflammatory mediators definitely have some role rather than a simple wear and tear, which affects cartilage, synovial membrane, ligaments, menisci and subchondral bone. ${ }^{4}$ There are numerous conservative treatments for Knee OA like NSAIDS (Non-steroidal anti-inflammatory drugs), opioids which are used to relieve pain but they only give short-term relief. 5 These treatments have systemic adverse effects, cause joint cartilage destruction and flare up of the osteoarthritic process. ${ }^{6}$ Other non-operative modalities like intra-articular injections of corticosteroids, ozone, viscosupplements (Hyaluronic acid) which are used to relieve pain have inconsistent results, are costly and need repeated injections. ${ }^{7,8,9}$ Also, they have limited effect on reducing chondrocyte degeneration and improving regeneration. ${ }^{10}$

In recent times, tissue healing has been taken into consideration to prevent progression of the disease. New studies have focused on modern therapeutic methods stimulating cartilage healing process and preventing its damage, including application of cytokinase inhibitors, matrix metalloproteinase inhibitors, stem cells, gene therapy and growth factors (GF). ${ }^{11}$ Among these, GFs have been studied in vitro and in vivo to have healing effect on the cartilage with promising results. ${ }^{12,13,14}$ Significant amount of GF is present in platelet alpha granules. ${ }^{15}$ Due to this, platelet rich plasma (PRP) application has emerged as a treatment option for OA. Platelet-rich plasma (PRP) is an autologous biologic treatment including patient's own plasma, containing growth factors released from platelets and endogenous fibrin scaffold. ${ }^{16}$ PRP includes plasma proteins that act as mesenchymal cell adhesion molecules like fibrin, fibronectin and vitronectin which appear during recovery process following a trauma in the human body and regulate antiinflammatory signals and equilibrate angiogenesis. ${ }^{17}$

Recent studies state that PRP injection in OA knee joint are promising for relieving pain, improving the range of motion of the knee joint and the overall quality of life. ${ }^{18,19}$ So, we have undertaken the study where we have used PRP for OA Knee joint in 100 patients and followed them up at 2,4,8 and 12 weeks.

\section{METHODS}

The study was approved by the Ethics Committee of R. G. Kar Medical College and Hospital. The study subjects were screened in the Pain Clinic OPD and the procedure was done in Pain Intervention OT of the Department of Anaesthesiology, R. G. Kar Medical College and Hospital, Kolkata, West Bengal from January 2016 to June 2016.
Patients with Knee Osteoarthritis (OA) Grade I, II and III Kellgren-Lawrence score based on American Rheumatology Criteria ${ }^{20,21}$ aged $30-80$ years, ASA 1,2 and 3 not responding to conservative treatment for more than three months, were considered for this prospective interventional study. Proper consents were taken in the patient's preferred language and the procedure was explained to the patient and their attendants. The study participants underwent proper history taking, physical examination, laboratory testing (Complete blood count with differential count, erythrocyte sedimentation rate (ESR), Platelet count, Blood Sugar for fasting, Uric acid, C-reactive protein (CRP)), knee radiography in standing position antero-posterior view and $30^{\circ}$ semi flexion latero-lateral view and survey of used medications and supplements.

Patients were excluded from the study if they had any of the following criteria: Kellgren-Lawrence score 4, age more than 80 years, any systemic disorders (Uncontrolled diabetes mellitus, rheumatoid arthritis, coagulopathy, severe cardiovascular diseases, infections, immunosuppression), poly-articular disease, major axial deviation (Varus $>20^{\circ}$, valgus $>20^{\circ}$ ), active wound in the knee area, anticoagulant medications 10 days before injection, history of intraarticular injections of corticosteroids or systemic corticosteroids in the last 3 weeks before injections, previous knee operations, pregnancy, patients with Haemoglobin < $10 \mathrm{~g} / \mathrm{dl}$ and platelet values less than $1,50,000$ and lastly, patient's refusal.

For all patients, Visual Analogue Scale (VAS) score for assessing pain intensity and Western Ontario and McMaster Universities Arthritis Index (WOMAC) questionnaire ${ }^{22}$ for assessing pain, stiffness and physical function of the joint were used before the procedure and 2,4,8 and 12 weeks after the procedure.

WOMAC questionnaire is recommended for OA studies by Outcome Measures in Rheumatology Clinical Trials, is a reliable and valid method for the assessment of patients with knee and hip 0A.22 It measures pain, stiffness, and physical function in patients with knee and hip osteoarthritis. The WOMAC questionnaire consists of 24 items divided into 3 subscales-

- Pain (5 Items)- During walking, using stairs, in bed, sitting or lying, and standing.

- Stiffness (2 Items)- After first waking and later in the day.

- Physical Function (17 Items)- Stair use, rising from sitting, standing, bending, walking, getting in/ out of a car, shopping, putting on/ taking off socks, rising from bed, lying in bed, getting in/ out of bath, sitting, getting on/ off toilet, heavy household duties, light household duties.

Each question is scored from 0 to 5 with fewer scores indicating less pain and better functional status. For WOMAC, the minimum score is zero and the maximum score, which represents the highest grade of debilitation, is 96. For the process of PRP preparation, the patients were referred to the Blood Bank of R. G. Kar Medical College. In order to prepare PRP, $50 \mathrm{~mL}$ of blood was first collected from the patient's upper limb cubital vein using an 18G needle, in which acid citrate dextrose solution was added as an anticoagulant. The blood sample underwent centrifugation in the Cryofuge 6000i machine at $2450 \mathrm{rpm}$ for 8 minutes for separation of the erythrocytes and the concentration of platelets. The final 
product was $10-20 \mathrm{ml}$ of PRP with 4 to 6 times baseline platelet count (Using cell counter) was sent to the Pain Intervention OT for intra articular injection.

The patient was placed in a supine position, the knee being slightly bent with the help of a popliteal cushion The superolateral approach was used for injection, which has been shown to be the safest, ensuring intra-articular penetration of the drug in up to $93 \%$ of cases. ${ }^{23}$ Under proper ultra-sound guidance and aseptic precautions, the needle was inserted at an angle of approximately $45^{\circ}$ toward the medial joint line of the knee until reaching the "soft spot" between the patella and the femur, next to the junction of the line going through the lateral patellar edge and the line going through the superior pole of the patella. Before the PRP was injected, the piston of the syringe was drawn back slightly to ensure that the needle was properly in the joint by free aspiration flow of synovial fluid after which $6 \mathrm{ml}$ of PRP was injected into the spot. After the technique, the patient's injected site was properly bandaged with elastocrepe. They were monitored for 30 minutes to ensure there were no adverse reactions. After that they were discharged and were advised to have rest for 24 to 48 hours and limit weightbearing on the injected joint. They were also instructed to use cold compression two times a day each time for 15 minutes. They were prescribed Paracetamol weight-wise for five days for pain but were strictly prohibited to take NSAIDs and any steroids. They were also advised mild to moderate Quadriceps exercise one week after injection and increase it as tolerated. The patients were then re-assessed after 2 weeks, 4 weeks, 8 weeks and 12 weeks using the VAS score and the WOMAC score.

\section{Statistical Analysis}

Statistical data was collected and analysed using appropriate statistical tool SPSS v23. Repeated measures ANOVA were used. $\mathrm{p}<0.05$ was considered to be significant.

\section{RESULTS}

Table 1, 2, 3 show that the demographic profile of the patient and were comparable in all the groups. Figure 1 and Table 4 showed pre procedural and post procedural VAS scores at 2 , 4,8 and 12 weeks. Post procedural VAS score was significantly less compared to pre procedure, $\mathrm{p}<0.01$ at $2,4,8$ and 12 weeks in the three groups. Grade I and II have significant improvement in VAS score compared to Grade III at the end of 12 weeks $p<0.01$. Figure 2 and Table 5 showed pre procedural and post procedural WOMAC scores at 2, 4, 8 and 12 weeks.

\begin{tabular}{|c|c|c|c|}
\hline & N & Mean & Standard Deviation \\
\hline Age (in years) & 100 & 53.910 & 8.4400 \\
\hline BMI (in $\left.\mathrm{kg} / \mathrm{m}^{2}\right)^{*}$ & 100 & 25.6579 & 3.69790 \\
\hline \multicolumn{3}{|c|}{ Table 1. Age and BMI of the Patients } \\
\hline *BMI: Body Mass Index
\end{tabular}

\begin{tabular}{|c|c|c|}
\hline Sex & Frequency & Percent \\
\hline Female & 69 & 69.0 \\
\hline Male & 31 & 31.0 \\
\hline Total & 100 & 100.0 \\
\hline \multicolumn{2}{|c|}{ Table 2. Sex Distribution of the Patients } \\
\hline
\end{tabular}

\begin{tabular}{|c|c|c|}
\hline OA Grade & Frequency & Percent \\
\hline 1 & 33 & 33.0 \\
\hline 2 & 36 & 36.0 \\
\hline 3 & 31 & 31.0 \\
\hline Total & $\mathbf{1 0 0}$ & $\mathbf{1 0 0 . 0}$ \\
\hline \multicolumn{2}{|c|}{ Table 3. Frequency of Grading of Osteoarthritis (OA) } \\
\multicolumn{2}{|l}{}
\end{tabular}

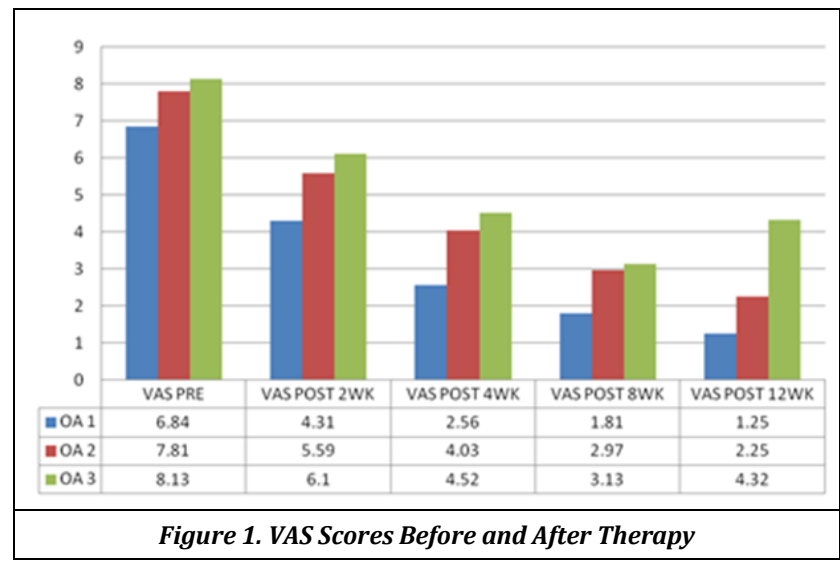

\begin{tabular}{|c|c|c|c|c|c|}
\hline & & Mean & S.D. & $\begin{array}{c}\text { Paired- } \\
\text { t test }\end{array}$ & p \\
\hline 1. & VAS PRE-VAS POST 2WK & & & & \\
\hline 2. & VAS POST 2WK-VAS POST 4WK & 1.750 & .718 & 13.780 & $<0.01$ \\
\hline 3. & VAS POST 4WK-VAS POST 8WK & .750 & .440 & 9.644 & $<0.01$ \\
\hline 4. & VAS POST 8WK-VAS POST 12WK & .563 & .564 & 5.638 & $<0.01$ \\
\hline \multicolumn{6}{|c|}{ Table 4. VAS Scores Before and After Therapy } \\
\hline
\end{tabular}

Table 4. VAS Scores Before and After Therapy

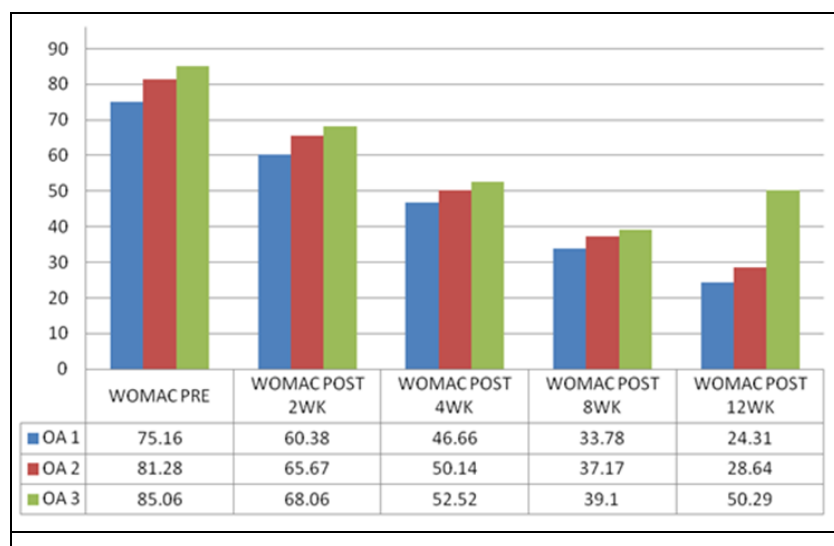

Figure 2. WOMAC Scores Before and After Therapy

\begin{tabular}{|c|c|c|c|c|c|}
\hline \multicolumn{2}{|c|}{} & \multicolumn{2}{|c|}{ Paired Differences } & Paired- \\
\cline { 3 - 6 } & Mean & S.D. & t test & p \\
\hline 1. & $\begin{array}{c}\text { WOMAC POST 2WK- } \\
\text { WOMAC POST 4WK }\end{array}$ & 13.719 & 4.357 & 17.812 & $<0.01$ \\
\hline 2. & $\begin{array}{c}\text { WOMAC POST 4WK - } \\
\text { WOMAC POST 8WK }\end{array}$ & 12.875 & 3.687 & 19.752 & $<0.01$ \\
\hline 3 & $\begin{array}{c}\text { WOMAC POST 8WK - } \\
\text { WOMAC POST 12WK }\end{array}$ & 9.469 & 4.579 & 11.698 & $<0.01$ \\
\hline \multicolumn{6}{|c|}{ Table 5. WOMAC Scores Before and After Therapy } \\
\hline
\end{tabular}

Post procedural WOMAC scores were significantly less compared to pre procedure, $\mathrm{p}<0.01$ at 2, 4, 8 and 12 weeks in the three group. Grade I and II have significant improvement in WOMAC score compared to Grade III at the end of 12 weeks, $\mathrm{p}<0.01$.

\section{DISCUSSION}

Osteoarthritis (OA) is a chronic disease defined by progressive degenerative changes of the joint and loss of cartilage on joint surfaces. The hallmark symptom of Knee OA is pain. But the presence and severity of joint pain correlate 
poorly with structural evidence of joint damage. The degeneration occurring in the joint leads to changes in the catabolic and anabolic activity of chondrocytes. As a result, other components of the joint get compromised which may lead to meniscus degeneration, sclerosis, bone deformity as well as intermittent synovial inflammation and sub-chondral tissue oedema. This disease affects the functional capacity and quality of life (QOL) in patients by producing pain, stiffness and limitation of range of motion of the joint. ${ }^{24}$

The aim of management of knee $\mathrm{OA}$ is to reduce pain and stiffness, protect or regain range of motion and muscle strength, and decrease dependence in daily living activities. Thus, in recent years, cartilage supporting or improving medications have been investigated. The action of Plateletrich Plasma is to release growth factors (GF) thereby causing healing and regeneration of cartilages. ${ }^{19} \mathrm{GF}$ increases the synthesis of chondrocyte matrix and also stimulates chondrogenic cell proliferation. ${ }^{25}$ It reduces the activation of nuclear factor kappa B which is known to have a vital role in the pathogenesis of $\mathrm{OA}$, by the inhibition of inflammatory process which is induced by interleukin-1 beta. ${ }^{26}$

Despite its wide clinical application, only a few reports have documented results of PRP in the treatment of knee joint OA.7,16,27 In 2008 Sánchez et al. published a retrospective interventional study involving 30 patients for PRP with encouraging results. ${ }^{16}$ In 2010 Sampson et al. published a study on 14 patients with clinical and radiographic signs of $\mathrm{OA}$ and previous unsuccessful conservative management who received 3 PRP injections at one month apart. Evaluation was carried out for 52 weeks. VAS was significantly improved at 1-year follow-up. ${ }^{7}$ Kon et al. reported 91 patients receiving PRP injections in one-week intervals. They noted that $80 \%$ of patients were satisfied with the treatment. ${ }^{28}$ This prospective interventional study was carried out on 100 Indian patients. The majority of the patients showed a decreasing trend of VAS and WOMAC scores from 2 weeks itself post-procedure.

Typically, pain relief starts to occur within three to four weeks and continues to improve over a period of three to six months following a PRP injection. ${ }^{29}$ The recovery time frame varies depending on what we are treating. Sometimes arthritic joints respond much faster to these injections than a patient being treated for tendonitis. ${ }^{31}$ Visual Analogue Score and WOMAC score was significantly improved in all the grades of Knee OA. Filardo et al. observed better improvement in less degenerated knee and worse outcome for older patients. ${ }^{30}$ In this study, Grade I and II showed significant improvement compared to Grade III at 12 weeks. We have observed no significant age or body weight related correlation in this small study group.

The patients did not describe long-term complications related to the procedure and no serious adverse events attributable to the treatment. The majority of the patients expressed overall satisfaction at 3 months after treatment. We have used only one injection in our cases because none of the patients complained of significant pain during 12 weeks observation period. Ayhan et al concluded even one PRP application is safe, effective and low-cost method. ${ }^{31}$ There is no guideline till date about the dose and frequency of PRP injections. 4 to $6 \mathrm{ml}$ of PRP was used in most of the previous studies $^{28}$ so we have used $6 \mathrm{ml}$ the higher dose with an idea to avoid repeated injections in patients.
There is no standardized technique for centrifugation but double centrifuge with more than 4 to 6 times platelet count from baseline gives encouraging results. ${ }^{25}$ In this study 2450 rpm was used for 8 minutes for separation of the erythrocytes and the concentration of platelets to 4 to 6 times of baseline.

The limitation of the study was the number of patients observed, which was too small to evaluate age and body weight related correlation in the result and the duration of the interventional study which was only 12 weeks. We have only assessed the short-term effect of PRP. Further studies are needed to confirm these results and understand the mechanism of action, and to find other application modalities, with different platelet and GF concentrations and injection timing, which provide better and more durable results. The positive trends and safety profile demonstrated could potentially be used to inspire a larger, blinded, and randomized clinical trial across different age groups and populations to determine whether platelet-rich plasma is safe and effective for the treatment of knee osteoarthritis.

\section{CONCLUSIONS}

Platelet-rich plasma therapy is safe and effective in relieving pain and improving functions of knee joint in patients suffering from knee osteoarthritis; particularly Grade I and Grade II (Kellgren-Lawrence score)

\section{REFERENCES}

[1] Lawrence RC, Felson DT, Helmick CG, et al. Estimates of the prevalence of arthritis and other rheumatic conditions in the United States Part II. Arthritis Rheum 2008;58(1):26-35.

[2] Neustadt DH. Osteoarthritis. In: Rakel RE, edr. Conn's Current therapy. Philadelphia, PA: W.B. Saunders 2003: p. 1075-9.

[3] Stewart WF, Ricci JA, Chee E, et al. Lost productive time and cost due to common pain conditions in the US workforce. JAMA 2003;290(18):2443-54.

[4] Martel-Pelletier J, Boileau C, Pelletier JP, et al. Cartilage in normal and osteoarthritis conditions. Best Pract Res Clin Rheumatol 2008;22(2):351-84.

[5] McArthur BA, Dy CJ, Fabricant PD, et al. Long term safety, efficacy and patient acceptability of hyaluronic acid injection in patients with painful osteoarthritis of the knee. Patient Prefer Adherence 2012;6:905-10.

[6] Kon E, Filardo G, Drobnic M, et al. Non-surgical management of early knee osteoarthritis. Knee Surg Sports Traumatol Arthrosc 2012;20(3):436-49.

[7] Sampson S, Gerhardt M, Mandelbaum B. Platelet rich plasma injection grafts for musculoskeletal injuries: a review. Curr Rev Musculoskelet Med 2008;1(3-4):16574.

[8] Oliver KS, Crane DM. Platelet rich plasma grafts in musculoskeletal medicine. Journal of Prolotherapy 2010;2(2):371-6. 
[9] Frizziero A, Giannotti E, Ferraro C, et al. Platelet rich plasma intra-articular injections: a new therapeutic strategy for the treatment of knee osteoarthritis in sport rehabilitation. A systematic review. Sport Sci Health 2012;8:15-22.

[10] Handl M, Amler E, Bräun K, et al. Positive effect of oral supplementation with glycosaminoglycans and antioxidants on the regeneration of osteochondral defects in the knee joint. Physiol Res 2007;56(2):243-9.

[11] Wang-Saegusa A, Cugat R, Ares O, et al. Infiltration of plasma rich in growth factors for osteoarthritis of the knee short-term effects on function and quality of life. Archives of Orthopaedic and Trauma Surgery 2011;131(3):311-7.

[12] Mishra A, Woodall J Jr, Vieira A. Treatment of tendon and muscle using platelet-rich plasma. Clinics in Sports Medicine 2009;28(1):113-25.

[13] Rabago D, Best TM, Zgierska AE, et al. A systematic review of four injection therapies for lateral epicondylosis: prolotherapy, polidocanol, whole blood and platelet-rich plasma. British Journal of Sports Medicine 2009;43(7):471-81.

[14] Bir SC, Esaki J, Marui A, et al. Angiogenic properties of sustained release platelet-rich plasma: characterization in-vitro and in the ischemic hind limb of the mouse. Journal of Vascular Surgery 2009;50(4):870-9.

[15] Cook JL, Anderson CC, Kreeger JM, et al. Effects of human recombinant interleukin - 1 beta on canine articular chondrocytes in three-dimensional culture. Am J Vet Res 2000;61(7):766-70.

[16] Sánchez M, Anitua E, Azofra J, et al. Intra-articular injection of an autologous preparation rich in growth factors for the treatment of knee OA: a retrospective cohort study. Clin Exp Rheumatol 2008;26(5):910-3.

[17] Kang YH, Jeon SH, Park JY, et al. Platelet rich fibrin is a Bioscaffold and reservoir of growth factors for tissue regeneration. Tissue Eng Part A 2011;17(3-4):349-59.

[18] Filardo G, Kon E, Buda R, et al. Platelet-rich plasma intraarticular knee injections for the treatment of degenerative cartilage lesions and osteoarthritis. Knee Surg Sports Traumatol Arthrosc 2011;19(4):528-35.

[19] Spakova T, Rosocha J, Lacko M, et al. Treatment of knee joint osteoarthritis with autologous platelet-rich plasma in comparison with hyaluronic acid. Am J Phys Med Rehabil 2012;91(5):411-7.

[20] Hochberg MC, Altman RD, April KT, et al. American College of Rheumatology 2012 recommendations for the use of non-pharmacologic and pharmacologic therapies in osteoarthritis of the hand, hip and knee. Arthritis Care Res (Hoboken) 2012;64(4):465-74.
[21] Misso ML, Pitt VJ, Jones KM, et al. Quality and consistency of clinical practice guidelines for diagnosis and management of osteoarthritis of the hip and knee: a descriptive overview of published guidelines. Med J Aust 2008;189(7):394-9.

[22] Bellamy N, Buchanan WW, Goldsmith $\mathrm{CH}$, et al. Validation study of WOMAC: a health status instrument for measuring clinically important patient relevant outcomes to antirheumatic drug therapy in patients with osteoarthritis of the hip or knee. J Rheumatol 1988;15(12):1833-40.

[23] Jackson DW, Evans NA, Thomas BM. Accuracy of needle placement into the intra-articular space of the knee. J Bone Joint Surg Am 2002;84(9):1522-7.

[24] Zhu Y, Yuan M, Meng HY, et al. Basic science and clinical application of platelet-rich plasma for cartilage defects and osteoarthritis: a review. Osteoarthritis Cartilage 2013;21(11):1627-37.

[25] Fortier LA, Barker JU, Strauss EJ, et al. The role of growth factors in cartilage repair. Clin Orthop Relat Res 2011;469(10):2706-15.

[26] Van Buul GM, Koevoet WL, Kops N, et al. Platelet-rich plasma releasate inhibits inflammatory processes in osteoarthritic chondrocytes. Am J Sports Med 2011;39(11):2362-70.

[27] Raeissadat SA, Sedighipour L, Rayegani SM, et al. Effect of platelet-rich plasma (PRP) versus autologous whole blood on pain and function improvement in tennis elbow: a randomized clinical trial. Pain Res Treat 2014;2014:191525.

[28] Kon E, Buda R, Filardo G, et al. Platelet-rich plasma: intra-articular knee injections produced favorable results on degenerative cartilage lesions. Knee Surg Sports Traumatol Arthrosc 2010;18(4):472-9.

[29] Bhattacharya D. Mystery of platelet rich plasma injection in painful conditions. Editorial. Indian J Pain 2015;29(3):121-3.

[30] Filardo G, Kon E, Di Martino A, et al. Platelet - rich plasma vs hyaluronic acid to treat knee degenerative pathology: study design and preliminary results of a randomized controlled trial. BMC Musculoskeletal Disorders 2012;13:229.

[31] Ayhan E, Kesmezacar H, Akgun I. Intraarticular injections (corticosteroid, hyaluronic acid, platelet rich plasma) for the knee osteoarthritis. World Journal of Orthopaedics 2014;5(3):351-61. 